\title{
Die Mathematik ist eine gar herrliche Wissenschaft
}

\section{Journal Article}

\section{Author(s):}

Stammbach, Urs

Publication date:

2005

Permanent link:

https://doi.org/10.3929/ethz-b-000422890

Rights / license:

In Copyright - Non-Commercial Use Permitted

Originally published in:

Mitteilungen der Deutschen Mathematiker-Vereinigung 13(4), https://doi.org/10.1515/dmvm-2005-0082 


\section{„Die Mathematik ist eine gar herrliche Wissenschaft"“ von Urs Stammbach}

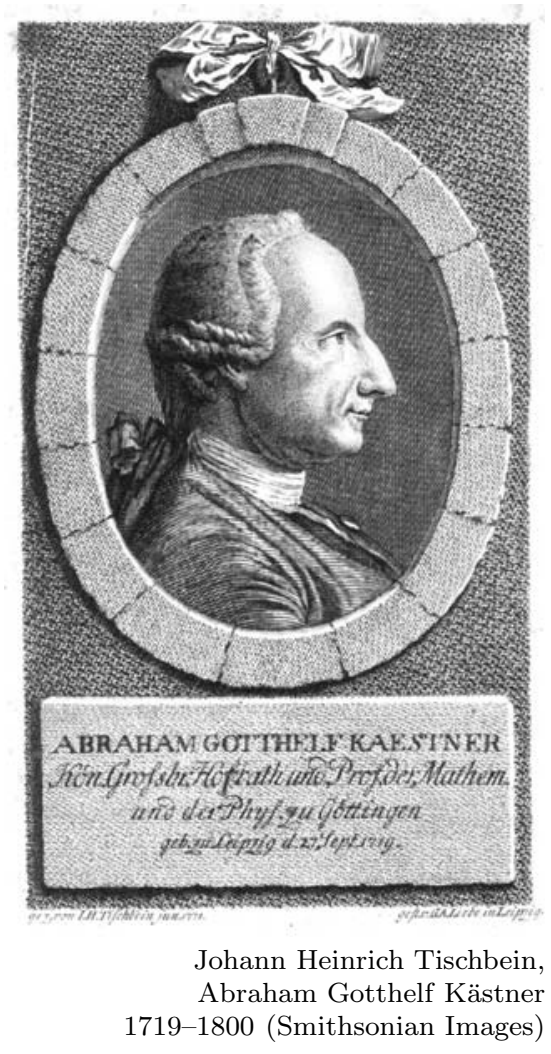

Ein Mathematiker, der einen Vortrag vor einem allgemeinen Publikum halten soll, steht vor einem schwierigen Problem. Wählt er ein Thema aus der Mathematik, so spaltet er die Zuhörerschaft automatisch in drei Teile: Ein Drittel, bestehend aus den Mit-Mathematikerinnen und Mathematikern wird nichts Neues zu hören bekommen und den Vortrag dementsprechend langweilig finden; ein zweites Drittel, bestehend aus mathematischen Laien wird den vertrackten mathematischen Gedankengängen nicht folgen können oder nicht folgen wollen und sich deswegen ebenfalls langweilen; nur das mittlere Drittel der Zuhörerschaft wird in einigen glücklichen Fällen dem Vortrag etwas Interessantes abgewinnen können.

Das Problem ist übrigens alt. Schon 1756 hatte der neuberufene Mathematikprofessor Abraham Gotthelf Kästner ${ }^{1}$ in seiner Antrittsrede in Göttingen damit zu kämpfen. Er sagte dort:

\section{Von wenigen und nur denen, die als verständig gelten, anerkannt zu werden, ist das Einzige, was ein Mathe- matiker [...] zu erwarten hat. Den Beifall der Menge zu finden, daran darf er gar nicht erst denken.}

Ich möchte hier dem Dilemma ausweichen; im Rahmen einer Abschiedsvorlesung darf man eine gewisse Narrenfreiheit beanspruchen. Ich will nicht über ein Thema der Mathematik sprechen, sondern ich möchte in diesem Vortrag einige Beispiele von Aussagen zusammenstellen, echte und solche aus der Literatur, in denen sich Nichtmathematiker über die Mathematik geäussert haben. Ich hoffe, damit nicht nur einen
Teil der Zuhörerschaft sondern alle Anwesenden anzusprechen und mit meinem Vortrag in den nächsten Minuten etwas unterhalten zu können. Die Zitate, die ich vorführen will, sind nicht zufällig aufgereiht, sondern sie sind locker eingebettet in eine historischgesellschaftliche Entwicklung, die auch für sich allein genommen interessant ist und Überraschungen enthält. Man möge mir verzeihen, wenn ab und zu dieser Hintergrund etwas zu prominent in Erscheinung treten sollte. Ganz zum Schluss werde ich dann noch einmal - und vom Vortragsthema etwas getrennt kurz auf das eben erwähnte Dilemma der Dreiteilung der Zuhörerschaft zurückkommen, die ich hier zu vermeiden versuche.

Ich beginne mit Arthur Schopenhauer (1788-1860), dem Philosophen. Es ist bekannt, dass Arthur Scho-

1 Abraham Gotthelf Kästner, 1719-1800, war noch in Göttingen tätig, als Carl Friedrich Gauss dort sein Studium aufnahm. Dieser sagte später einmal über Kästner, er sei der erste Mathematiker unter den Dichtern und der erste Dichter unter den Mathematikern gewesen. Diese Aussage ist vielleicht nicht so abwertend, wie es den Anschein macht: Käster hat nicht nur mehrere umfangreiche und häufig verwendete mathematische Lehrbücher geschrieben, sondern er hat daneben eine Vielzahl von launischen, kleinen Gedichten verfasst, in denen er manchmal auch die Mathematik zum Thema machte. 
penhauer nicht gut auf die Mathematik zu sprechen war. In seinen Schriften äusserte er seine Meinung mit aller wünschenswerten Deutlichkeit:

Dass die niedrigste aller Geistestätigkeit die arithmetische sei, wird dadurch belegt, dass sie die einzige ist, welche auch durch eine Maschine ausgeführt werden kann. [...] Nun läuft alle analysis finitorum et infinitorum im Grunde doch auf Rechnen zurück. Danach bemesse man den „mathematischen Tiefsinn,“ [. . .]

Rechnungen haben bloss Wert für die Praxis, nicht für die Theorie. Sogar kann man sagen, wo das Rechnen anfängt, hört das Verstehen auf.

Als Nichtmathematiker konnte Schopenhauer seine Meinung wohl äussern, sie blieb aber die Meinung eines Mathematik-Laien. Wohl aus diesem Grund versuchte er, stützende Zitate von Personen beizubringen, die dem Fache näher standen. Man spürt förmlich, wie es Schopenhauer Freude machte, derartige Aussagen aus der Literatur zusammenzutragen und genüsslich zu zitieren. So nahm er ganz offensichtlich gerne Bezug auf eine (ziemlich obskure) Veröffentlichung von William Hamilton, Professor für Logik und Metaphysik an der Universität in Edinburgh. ${ }^{2}$ Dieser hatte sich unter dem Titel Thoughts on the study of mathematics as a part of a liberal education Gedanken über den Wert der Mathematik als Unterrichtsfach gemacht. Hamilton hat darin die Ansicht festgehalten - und Schopenhauer zitierte sie mit unverhohlener Freude -, dass der einzige unmittelbare Nutzen der Mathematik darin bestehe,

dass sie unstäte und flatterhafte Köpfe gewöhnen kann, ihre Aufmerksamkeit zu fixieren.

Auch in den Werken von Georg Christoph Lichtenberg $^{3}$ glaubte er, weitere entsprechende Unterstützung gefunden $\mathrm{zu}$ haben. Lichtenberg war ein damals sehr bekannter, breit gebildeter Göttinger Physiker. Schopenhauer durfte annehmen, dass er auch als Fachmann für die Mathematik gelten konnte. Als einem Briefpartner Goethes brachte man Lichtenberg in der geisteswissenschaftlichen Welt ebenfalls Anerkennung entgegen. Seine Sammlung von Aphorismen, seine von ihm so genannten Sudelbücher, enthalten Sprüche zu sämtlichen Lebenslagen. Sie zeugen von einem sehr ausgeprägten Sinn für Humor, Ironie und Zynismus. Das Zitat, das Schopenhauer darin fand und in sein Werk aufnahm, lautete:

Es ist fast mit der Mathematik, wie mit der Theologie. Sowie die der letzteren Beflissenen, zumal wenn sie in Ämtern stehen, Anspruch auf einen besonderen Kredit von Heiligkeit und eine nähere Verwandtschaft mit Gott machen, obgleich sehr viele darunter wahre

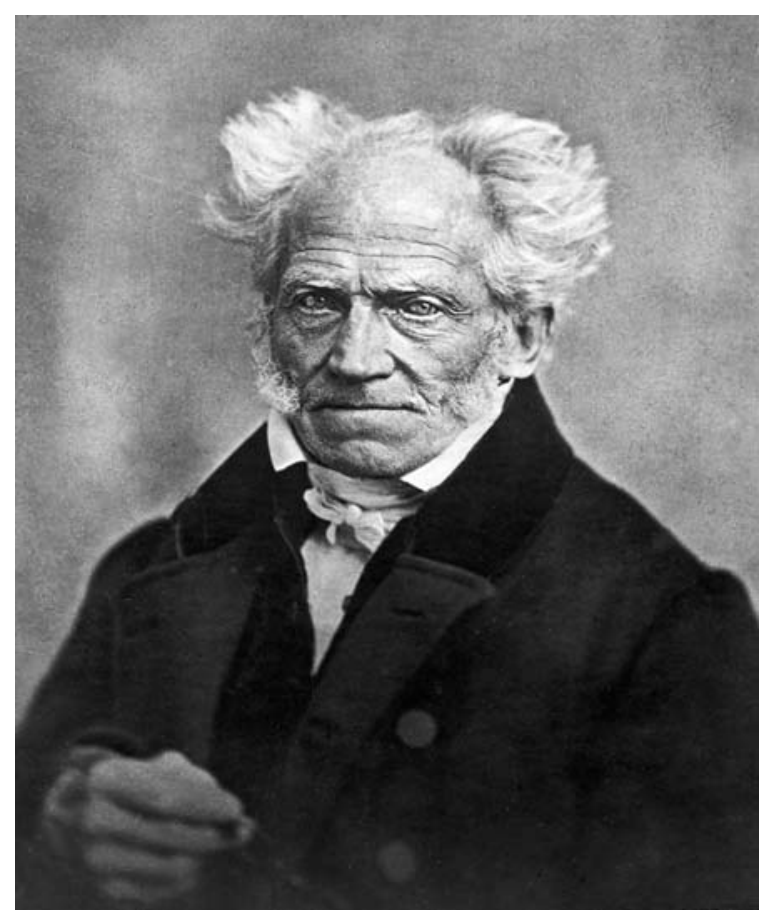

Arthur Schopenhauer, um 1858 (Bildarchiv Preußischer Kulturbesitz)

Taugenichtse sind, so verlangt sehr oft der sogenannte Mathematiker, für einen tiefen Denker gehalten zu werden, ob es gleich darunter die grössten Plunderköpfe gibt, die man nur finden kann, untauglich zu irgend einem Geschäft, das Nachdenken erfordert, wenn es nicht unmittelbar durch jene leichte Verbindungen von Zeichen geschehen kann, die mehr das Werk der Routine als des Denkens sind.

Schopenhauers Ansichten hatten bekanntlich auf das wissenschaftliche Leben in Deutschland grossen Einfluss. So wurde in den Auseinandersetzungen um eine Reform der höheren Schulen gegen Ende des 19. Jahrhunderts von der dezidierten Meinung Schopenhauers gerne Gebrauch gemacht, als es darum ging, den Anteil der Mathematik und der Naturwissenschaften im Programm der Gymnasialstufe festzulegen. Es war den Vertretern sprachlich-historischer Richtung ein Leichtes, damit ihre vorgefasste Meinung zu untermauern, die Meinung, die sie eindeutig und spitz im Ausspruch zusammenfassten: Mathematicus non est collega. Natürlich war es für die andere Seite einigermassen schwierig, sich gegen eine anerkannte Autorität durchzusetzen, wie es Schopenhauer damals immer noch war; in solchen Umständen ist ja, wie Sie wissen, die Qualität der Argumente jeweils zweitrangig.

2 William Hamilton, 1788-1856; nicht identisch mit Wiliam Rowan Hamilton, 1805-1865, der Mathematikern und Physikern durch die Entdeckung der Quaternionen, der Hamiltonschen Gleichungen und des Hamiltonschen Prinzipes wohlbekannt ist.

3 1742-1799. Carl Friedrich Gauss hörte bei seinem Studium in Göttingen noch bei Lichtenberg Vorlesungen. 


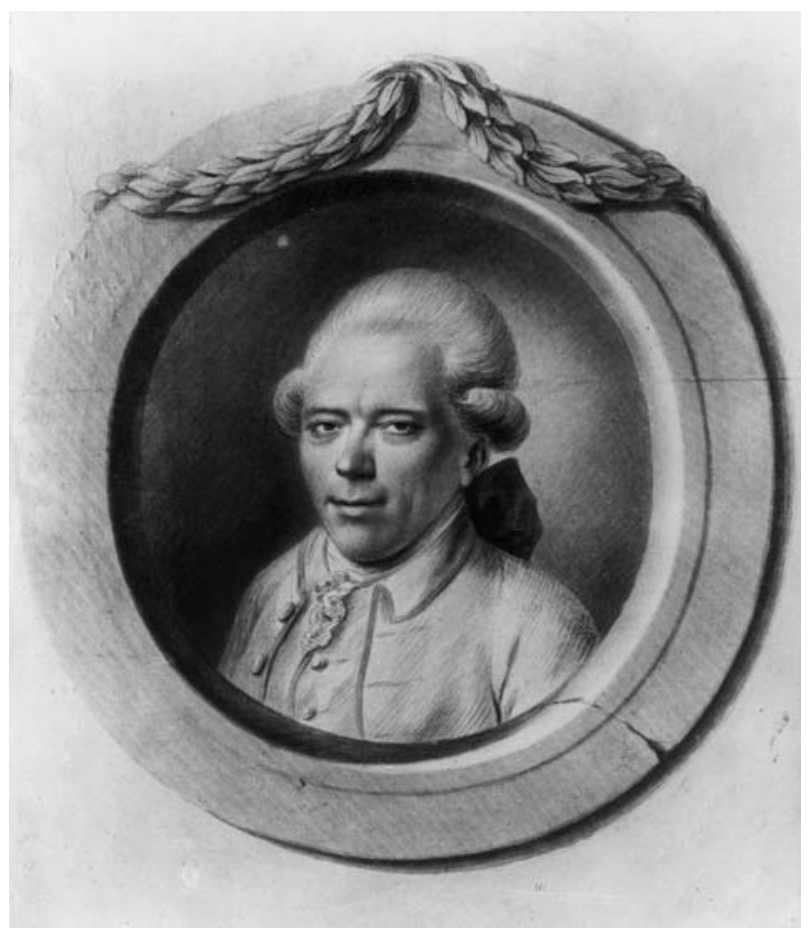

Ludwig Strecker, Georg Christoph Lichtenberg (1742-1799) (Bildarchiv Preußischer Kulturbesitz)

Kraftvoll zu Wort meldete sich zu dieser Kontroverse Alfred Pringsheim, ${ }^{4}$ Ordinarius für Mathematik an der Universität München. In einem Vortrag Über Wert und angeblichen Unwert der Mathematik ${ }^{5}$ anlässlich der Feier des 145. Stiftungstages der Königlich-Bayrischen Akademie im Jahre 1904 unterzog er die Auffassung Schopenhauers einer detaillierten Kritik. Ich will hier auf die einzelnen Punkte nicht eingehen, welche die Mathematik selbst betreffen, obschon sie durchaus Aufmerksamkeit verdienen, sondern ich beschränke mich auf die Ausführungen Pringsheims zum Lichtenberg Zitat. Hier wirft Pringsheim dem Philosophen eine plumpe und bösartige Fälschung vor. Schopenhauer habe nur den zweiten Teil des Aphorismus von Lichtenberg zitiert und damit die Aussage völlig verfälscht. In der Tat hatte Schopenhauer den ersten Satz weggelassen:

Die Mathematik ist eine gar herrliche Wissenschaft, aber die Mathematiker taugen oft den Henker nicht.
Es ist offensichtlich, dass das vollständige Lichtenberg Zitat einen ganz anderen Inhalt besitzt, als ihm Schopenhauer unterlegt hat. Es war vor allem auf einzelne Vertreter des Faches Mathematik gemünzt und überhaupt nicht auf die Mathematik als solche. Es wäre in der vollständigen Form weder gegen die Mathematik zu verwenden gewesen, noch hätte es den Jüngern Schopenhauers in ihrem Kampf gegen die Mathematik als Unterrichtsfach Nutzen gebracht. Es besteht wohl kein Zweifel, dass Schopenhauer in dieser Sache seine Leser bewusst irregeleitet hat. - Auch ich habe, wie Sie jetzt festgestellt haben, mit dem Titel meiner Vorlesung eine kleine Irreführung betrieben. Aber mit Blick auf meine Mitmathematiker wagte ich es schlicht nicht, auch den zweiten Teil des einleitenden Lichtenbergsatzes im Titel meiner Abschiedsvorlesung unterzubringen.

Die Meinung Lichtenbergs war in der Tat eine ganz andere, als sie Schopenhauer darstellte. An anderer Stelle seiner Sudelbücher sagte er zum Beispiel über die Mathematik:

Ich glaube doch auch, dass es im strengsten Verstand, für den Menschen nur eine einzige Wissenschaft gibt, und dieses ist reine Mathematik.

Klar, dass Schopenhauer diese Aussage Lichtenbergs nicht zitieren wollte, das hätte seinen Zwecken nicht gedient.

Alfred Pringsheim war damals in München eine prominente Persönlichkeit. Aus einer sehr reichen Familie stammend - sein Vater hatte in den Gründerjahren im Eisenbahngeschäft ein immenses Vermögen gemacht - hatte er für sich und seine Familie ein palastähnliches Haus an der Arcisstrasse bauen lassen. ${ }^{6}$ Sein Heim galt als ein Zentrum der Kultur im damaligen München. Pringsheim war ein grosser Freund der Musik Richard Wagners und mit diesem auch persönlich bekannt. ${ }^{7}$ Er stellte Klavierauszüge von Wagners Opern her, die er auf den beiden Konzertflügeln in seinen Haus den Gästen vorspielte beziehungsweise vorspielen liess. Die Maler Franz von Lenbach ${ }^{8}$ und Friedrich August von Kaulbach ${ }^{9}$ waren häufige Gäste im Hause Pringsheim. Sowohl Lenbach wie auch Kaulbach haben Mitglieder der

4 Geb. 2.9.1850 in Ohlau, gest. 25.6.1941 in Zürich. Ab 1871 Privatdozent, ab 1886 a.o. Professor und ab 1901 o. Professor in München; Emiritierung 1922. Arbeitete über Potenzreihen und Geschichte der Mathematik.

5 Alfred Pringsheim: Über Wert und angeblichen Unwert der Mathematik, Verlag der K.B. Akademie, 1904.

6 Baujahr 1889. Siehe dazu und zum Folgenden: Hanno-Walter Kruft: Alfred Pringsheim, Hans Thoma, Thomas Mann. Eine Münchner Konstellation. Abhandlungen der Bayrischen Akademie der Wissenschaften, Neue Folge, Heft $107,1993$.

7 Richard Wagner hatte in München im selben Viertel gewohnt, war aber bereits weggezogen, als Pringsheim seine Villa bauen liess.

8 1836-1904. Das feudale Wohnhaus Lenbachs befand sich nur eine Strassenecke von der Villa Pringsheim entfernt; es ist heute ein Museum (Lenbachhaus).

9 1850-1920.

10 Siehe Marianne Krüll: Im Netz der Zauberer, Bild 54; Katia Mann: Meine ungeschriebenen Memoiren, Fischer Verlag 1974, Bild von Kaulbach im Innern; Inge und Walter Jens: Frau Thomas Mann, verschiedene Bilder.

11 Björnstjerne Björnson, Nobelpreisträger für Literatur 1903. 


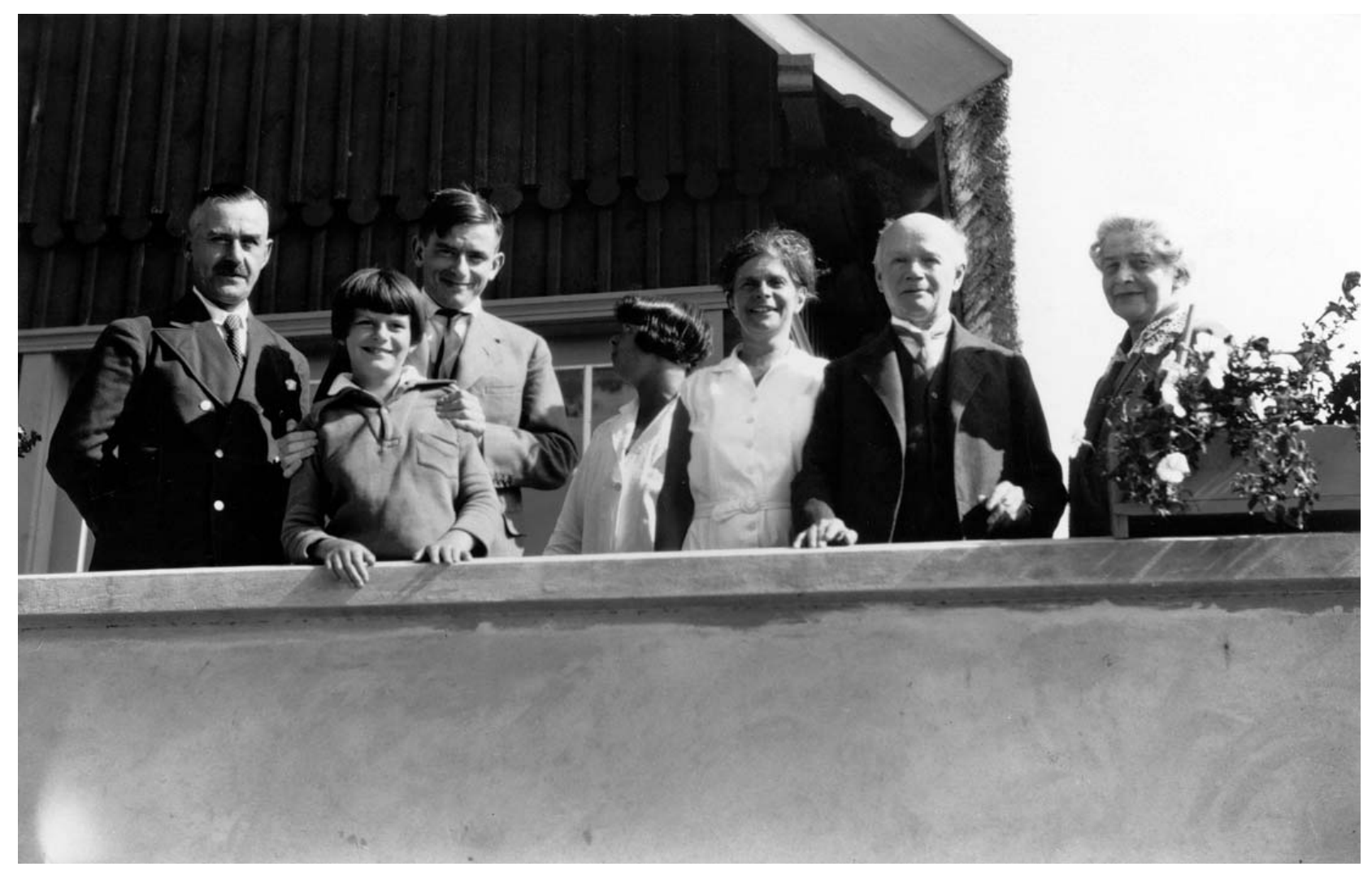

Thomas Mann, Elisabeth Mann, Golo Mann, Monika Mann, Katia Mann, Alfred Pringsheim und Hedwig Pringsheim am 2. September 1930 in Nidden (v.l.n.r.) (Archiv S. Fischer Verlag)

Familie porträtiert. ${ }^{10}$ Die Nobelpreisträger für Literatur, der Norweger Björnstjerne Björnson ${ }^{11}$ und der Deutsche Paul Heyse ${ }^{12}$ und die anderen Persönlichkeiten des kulturellen Münchens verkehrten ebenfalls in diesem Haus, in dem fast allabendlich Einladungen stattfanden. Die kostbare Sammlung Pringsheims von Renaissance-Majolika war weit über die Grenzen Münchens, ja Deutschlands bekannt. Von den drei Söhnen von Alfred Pringsheim studierte einer Physik, seine einzige Tochter studierte Mathematik.

Es muss kurz vor dem Vortrag von Alfred Pringsheim vor der Königlich-Bayrischen Akademie gewesen sein, als der damals in München lebende Thomas Mann auf ebendiese Tochter, auf die schöne Mathematikstudentin Katia Pringsheim aufmerksam wurde. Er hatte kurze Zeit vorher die Buddenbrooks herausgegeben und war dadurch rasch in ganz Deutschland bekannt geworden. Er wusste es einzurichten, dass er bei Pringsheims eingeladen wurde. Schon im Herbst 1903 schreibt er an seinen Freund Otto Grautoff über seinen Seelenzustand Folgendes: ${ }^{13}$

Wenn Du wüsstest, was für Wunder und wilde Mären ich in diesen Tagen - und Nächten - habe träumen lassen. Ich Narr! Ich Geck! der besser thäte, sich auf die Hosen zu setzen und etwas Gutes zu arbeiten, statt solchen Zaubermärchen nachzuhangen!

Während der Vater Pringsheim Thomas Mann eher etwas skeptisch zu begegnen schien, fand dieser in seiner Werbung um Katia bei der Mutter Unterstützung. ${ }^{14}$ Viel mehr als der Vater vertrat sie in der

12 Paul Heyse, Träger des Maximilianordens in München, erhielt 1910 als erster deutschsprachiger Dichter den Literaturnobelpreis, obschon er von einigen als Vielschreiber abgetan wurde. Gottfried Keller stand mit ihm in regem Briefwechsel. Heyse schlug Gottfried Keller für den Maximiliansorden vor, dieser antwortete aber trocken, ihm sei als Staatsschreiber die Annahme ausländischer Orden untersagt.

13 Siehe Nachwort von Hans Wysling zu Königliche Hoheit, Manesse Verlag, S. 484, Brief von Thomas Mann an Otto Grautoff vom 29. August 1903.

14 Die Mutter Katias, Hedwig Pringsheim-Dohm (1855-1942), war die Tochter des Berliner Schriftstellers Ernst Dohm (1819-83), des Mitbegründers und langjährigen Redakteurs des Kladderadatsch. Pringsheim hatte sie als junge Schauspielerin in Berlin kennengelernt und sie 1878 geheiratet. Ihre Mutter, Hedwig Dohm-Schlee (1833-1919), hatte eine führende Rolle in der deutschen Frauen-Emanzipationsbewegung gespielt. (Die Informationen stammen aus Hans-Rudolf Wiedemann: Thomas Manns Schwiegermutter erzählt, Verlag Graphische Werkstätten Lübeck, 3. Auflage 1988.) 


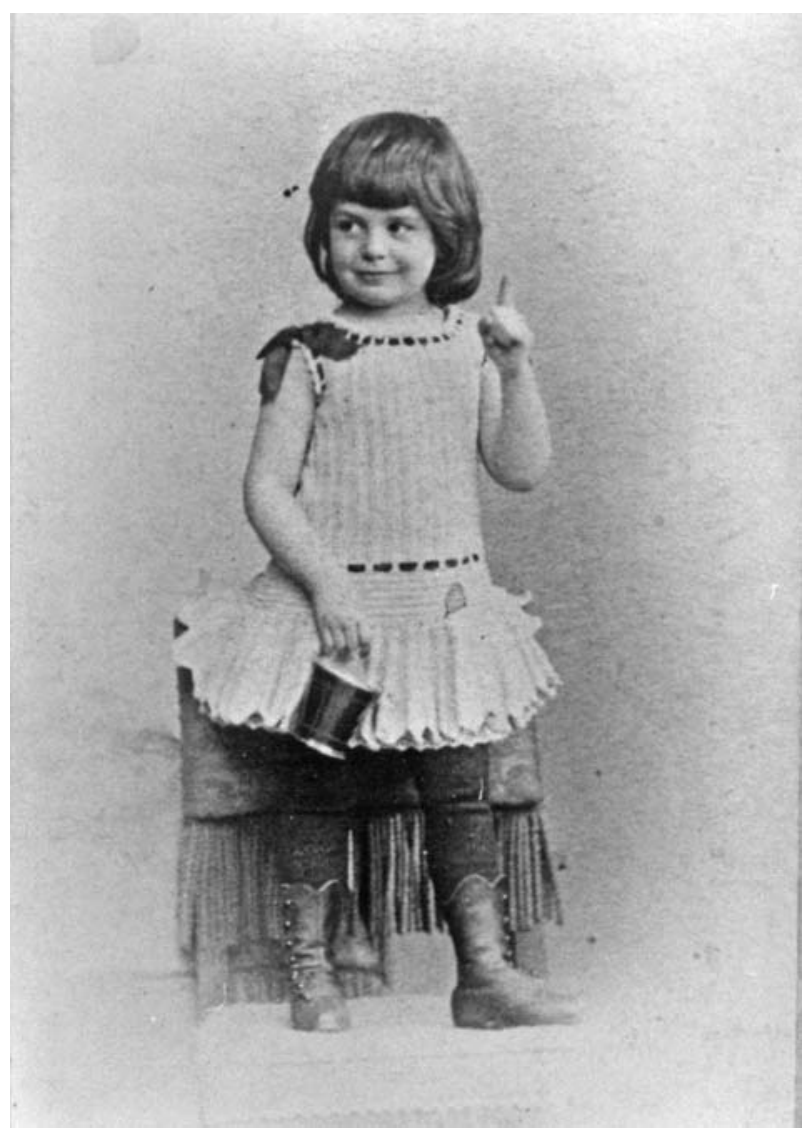

Katia Pringsheim, Portrait 1887

(Archiv S. Fischer Verlag)

Familie das literarische Interesse, war doch ihre Mutter, also die Grossmutter Katias, schriftstellerisch tä- tig gewesen und Autorin mehrerer Romane. ${ }^{15}$ Thomas Manns Werbung hatte Erfolg: die Heirat mit der 21jährigen Mathematikstudentin Katia Pringsheim fand im Februar 1905 statt. ${ }^{16}$

Thomas Mann hat bald nach der Heirat im Roman Königliche Hoheit ${ }^{17}$ Elemente der Erfahrungen verwendet, die er mit Katia und der Familie Pringsheim gesammelt hatte. Katia Mann, die Imma Spoelmann des Romans, meint zwar in ihren Lebenserinnerungen ${ }^{18}$, ihr eigenes Wesen entspreche nicht sehr der Romanfigur, räumt aber ein, dass verschiedene Begebenheiten zwischen ihr und Thomas Mann im Roman in nur leicht verfremdeter Form verarbeitet seien. Im Gegensatz zu ihr sei ihr Vater aber sehr gut in den Zügen des ausserordentlich reichen und etwas cholerisch veranlagten Dr. Samuel N. Spoelmanns wiederzuerkennen. ${ }^{19}$ Nicht ganz überraschend ist, dass Thomas Mann im Roman für sich selbst die Rolle eines Prinzen, des Prinzen Klaus Heinrich vorgesehen hat. In der Tat hatte er in Briefen sich und Katia vorher schon als Prinz und Prinzessin bezeichnet.

Es ist nicht allzu bekannt - auch in Mathematikkreisen nicht -, dass an einigen Stellen in diesem Roman die Mathematik auf sehr hübsche Weise zur Sprache kommt. Während einer ihrer ersten Begegnungen ergibt sich zum Beispiel zwischen Imma Spoelmann, alias Katia Pringsheim, und Klaus Heinrich, alias Thomas Mann der folgende Wortwechsel:

"Und Ihr Studium", fragte er, "gnädiges Fräulein? Darf ich mich erkundigen? Mathematik, wie ich weiss. Es strengt Sie nicht an? Ist es nicht furchtbar hart für

15 In seinem um 1930 geschriebenen Lebensabriss sagt Thomas Mann: „Von hier [dem Salon der Dichterin Ernst Rosmer] ging der Weg in das Pringheimsche Haus in der Arcisstrasse, ein Zentrum des Münchener gesellschaftlich-künstlerischen Lebens zur Zeit Ludwig II. und der Regentschaft, der Epoche Lenbachs, dessen pompöser Bestattungsfeier ich beigewohnt hatte. Die Atmosphäre des grossen Familienhauses, die mir die Umstände meiner Kindheit vergegenwärtigte, bezauberte mich. Das im Geiste kaufmännischer Kultureleganz Vertraute fand ich hier ins Prunkhaft-Künstlerische und Literarische mondänisiert und vergeistigt. Jedes der fünf erwachsenen Kinder (es waren fünf wie bei uns, die Jüngsten ein Zwillingspaar) besass eine eigene schöngebundene Bibliothek, zu schweigen von der reichen Kunst- und Musikbücherei des Hausherrn, eines der frühesten Wagnerianer, der den Meister gekannt hatte und nur aus einer Art von intelligenter Selbstbezwingung sich nicht ganz der Musik, sondern der Mathematik, die er dozierte, gewidmet hatte. Die Hausfrau, aus Berliner literarischem Haus stammend, Ernst und Hedwig Dohms Tochter, voller Sinn für meine Existenz und meine jugendliche Leistung, war der leidenschaftlichen Neigung nicht entgegen, die für die einzige Tochter des Hauses in mir keimte, und die von irgend jemand zu verbergen meine Einsamkeit mich nicht gelernt hatte. Ein grosses Ballfest in den goldnen Hochrenaissance-Räumen des Pringheimschen Hauses, eine glänzende und menschenreiche Veranstaltung, bei der ich vielleicht zum erstenmal die Sonne der öffentlichen Gunst und Achtung voll auf mir ruhen fühlte, brachte Gefühle zur Reife, auf die mein Leben zu gründen ich hoffen durfte."

16 Hochzeitstag war der 11. Februar 1905. Die Hochzeitsreise führte in die Schweiz, nach Zürich, wo das Paar im Hotel Baur au Lac logierte. In den unmittelbar folgenden Jahren kamen in rascher Folge vier Kinder (Erika 1905, Klaus 1906, Golo 1909, Monika 1910) zur Welt, dann nach einer 8jährigen Pause das fünfte und das sechste (Elisabeth 1918, Michael 1919). In einem Brief vom 7. September 1918 an ihre Freundin Dagny Björnson-Langen (1876-1974), zweite Tochter des oben erwähnten Björnson, äussert sich Katias Mutter, Hedwig Pringsheim-Dohm: Jemand hat gesagt, Th. M. scheine fruchtbarer in der Erzeugung von Kindern als von Büchern. - Die Briefe von Hedwig Pringsheim an ihre Freundin enthalten eine Reihe weiterer bemerkenswerter Aussagen, am 26. Februar 1921 etwa eine Bemerkung zu Ernst Jüngers Text In Stahlgewittern: „Ach Gott, Dagny, 'Schafsköpfe' ist wol ein mildes Wort, für die Herrschaften, die da wänten, aus dem 'Stalbad des Krieges' werde die Welt verschönt, verjüngt, erfrischt hervorgehen. Das sind ja Idioten, Wahnsinnige, Verbrecher." (Hedwig Pringsheim kümmerte sich in ihren Briefen nicht um die Dehnungs $h$.)

17 Entstanden 1905-1909, veröffentlicht 1909.

18 Katia Mann: Meine ungeschriebenen Memoiren, Fischer Verlag 1974.

19 Hedwig Pringsheim nannte in ihren Briefen an Dagny ihren Mann mehrmals den furchtbar süssen kleinen Mann, er sei, so am 7.September 1918, ein rechter Mummelgreis geworden, aber gesund dabei, frisch und immer verliebt. 
den Kopf?"

"Gar nicht", sagte sie. "Ich weiss nichts Hübscheres. Man spielt in den Lüften, sozusagen, oder schon ausserhalb der Luft, in staubfreier Gegend jedenfalls." ${ }^{20}$

Die Beziehungen zwischen dem Prinzen Klaus Heinrich und der Mathematikstudentin Imma Spoelmann werden mit der Zeit trotz der unterschiedlichen Herkunft zunehmend enger. Bei Besuchen und bei gemeinsamen Unternehmungen lernen sich die beiden näher kennen. Im Roman vereinbaren sie standesgemäss unter anderem einen gemeinsamen Ausritt, der am nächsten Tag mit schönem Wetter stattfinden soll. Als der Prinz Klaus Heinrich bei Spoelmanns die Tochter Imma dazu abholen will, entwickelt sich folgendes Gespräch:

"Nein", rief er [Klaus Heinrich], "heute dürfen Sie keine Algebra treiben, oder im luftleeren Raum spielen, wie Sie es nennen! Sehen Sie doch die Sonne! ... Darf ich ...?" Und er trat zum Tischchen und nahm das Kollegheft zur Hand.

Was er sah, war sinnverwirrend.

In einer krausen, kindlich dick aufgetragenen Schrift, die Imma Spoelmanns besondere Federhaltung erkennen liess, bedeckte ein phantastischer Hokuspokus, ein Hexensabbat verschränkter Runen die Seiten. Griechische Schriftzeichen waren mit lateinischen und mit Ziffern in verschiedener Höhe verkoppelt, mit Kreuzen und Strichen durchsetzt, ober- und unterhalb waagrechter Linien bruchartig aufgereiht, durch andere Linien zeltartig überdacht, durch Doppelstrichelchen gleichgewertet, durch runde Klammern zu grossen Formelmassen vereinigt. Einzelne Buchstaben, wie Schildwachen vorgeschoben, waren rechts oberhalb der umklammerten Gruppen ausgesetzt. Kabbalistische Male, vollständig unverständlich dem Laiensinn, umfassten mit ihren Armen Buchstaben und Zahlen, während Zahlenbrüche ihnen voranstanden und Zahlen und Buchstaben ihnen zu Häupten und Füssen schwebten. Sonderbare Silben, Abkürzungen geheimnisvoller Worte waren überall eingestreut, und zwischen den nekromantischen Kolonnen standen geschriebene Sätze und Bemerkungen in täglicher Sprache, deren Sinn so hoch über allen menschlichen Dingen war, dass man sie lesen konnte, ohne mehr davon $\mathrm{zu}$ verstehen, als von einem Zaubergemurmel.

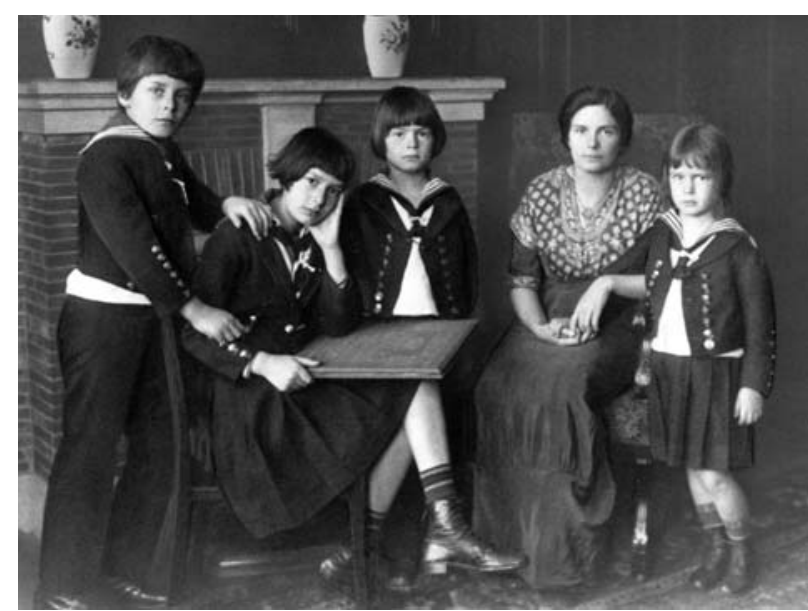

Katia Mann, mit Klaus, Erika, Golo und Monika, 1915 (Archiv S. Fischer Verlag)

Thomas Mann lässt seinen Klaus Heinrich nach dem Blick in Immas Kollegheft so reagieren wie es Nichtmathematiker in solchen Fällen üblicherweise tun. Klaus Heinrich wandte sich zu Imma Spoelmann, wunderte sich, dass in deren Köpfchen dies alles Sinn und hohes, spielendes Leben hatte und sagt:

"Und über diesen gottlosen Künsten wollen Sie den schönen Vormittag versäumen?"

Wer den Roman Königliche Hoheit kennt, erinnert sich sicher an die zahlreichen, reizvollen Stellen, in denen die Begegnungen des sehr abgeschirmt erzogenen Prinzen Klaus Heinrich mit der quirligen und sich mit amerikanischer Unbekümmertheit bewegenden Imma Spoelmann geschildert sind. Eine dieser Stellen wollen wir noch ansehen, denn sie nimmt nicht nur direkt auf die Mathematik Bezug, sondern sie trägt auch durchaus modernen Charakter. Nach seinem Amtsantritt hatte Klaus Heinrich von den gravierenden finanziellen Schwierigkeiten seines Staates erfahren. Er wollte sich selbst ein Bild über die Situation machen und begann, Bücher über Finanzwissenschaft zu studieren. Um den wissenschaftlichen, oft wohl auch mit etwas Mathematik vermischten Gedankengängen folgen zu können, bittet er Imma Spoelmann - die Mathematikerin! - um Hilfe. Ihre Antwort nach der Lektüre der Werke lautete:

"Es ist leicht!" sagte sie und sah lachend auf. "Mich nimmt wunder, dass es im Grunde so einfach ist. Algebra ist viel schwerer, Prinz ..."

Im Roman rettete am Schluss nicht die Finanzmathematik das verschuldete Königtum vor dem Bankrott,

20 Man weiss, dass sich Thomas Mann vor der Niederschrift seiner Werke jeweils in allen Einzelheiten vorbereitet hat. So ist anzunehmen, dass ihm der Vortrag seines Schwiegervaters sehr gut bekannt gewesen ist. Dort hatte Pringsheim festgestellt - die Parallelen sind offensichtlich: „In dem wahren Mathematiker steckt allemal ein gutes Stück vom Künstler: vom Architekten, ja vom Poeten. Ausserhalb der realen Welt, doch in erkennbarem Zusammenhang mit ihr, haben die Mathematiker in schöpferischer Gedankenarbeit sich eine ideale erbaut, die sie zur vollkommensten aller Welten auszugestalten suchen und nach allen Richtungen durchforsten." 
sondern die Mitgift, welche die reiche Imma Spoelmann in die Ehe mitbrachte. Mag sein, dass es heute umgekehrt wäre!

Zurück zu Schopenhauer. Über ihn hatte Thomas Mann bekanntlich andere Ansichten, als sie Alfred Pringsheim in seiner Rede vor der Bayrischen Akademie vertreten hatte. Er hatte Schopenhauer intensiv studiert, die Lektüre Schopenhauers war für ihn ${ }^{21}$ ein seelisches Erlebnis ersten Ranges und unvergesslicher Art, und in späteren Jahren gab er sogar eine gekürzte Fassung von Die Welt als Wille und Vorstellung heraus und versah sie mit einer längeren Einleitung. Es ist nicht verwunderlich, dass es zwischen Alfred Pringsheim und Thomas Mann bei diesem Thema zu Meinungsverschiedenheiten kam. Golo Mann, der Sohn von Thomas Mann, berichtet in seinen Lebenserinnerungen ${ }^{22}$ über ein Silvesterfest im Hause Pringsheim, an dem er als Jüngling teilgenommen hatte und an dem Schopenhauer unglücklicherweise zum Gesprächsthema wurde. Alfred und sein Sohn Peter, der Physiker, hätten mit Verachtung von Schopenhauers Werken gesprochen, es sei nichts als unbeweisbare Träumerei und Geschwätz. Golo Mann erzählt:

TM, beim Anhören solcher Reden wurde blass vor Zorn; er hielt sie für eine gezielte Beleidigung. Sie [die Pringsheims] mussten doch wissen, was Schopenhauer für ihn bedeutete [...]. Mit bebender Stimme zitierte er Nietzsche;

Was er lehrte, ist abgethan

Was er lebte, bleibt bestahn:

Seht ihn nur an!

Niemandem war er unterthan!

Danach verstummte das Gespräch; nach ein paar für mich fürchterlichen Minuten trennte man sich. Es war das letzte Silvesterfest im Hause Pringsheim.

Die Auseinandersetzung muss tatsächlich ziemlich heftig gewesen sein, denn Katia Mann hat das grässliche malentendu, wie sie es nennt, kurz darauf in einem Brief an ihre Tochter Erika ${ }^{23}$ beschrieben, und mitgeteilt, dass ihr Mann zuhause einen förmlichen Nervenzusammenbruch erlitten habe. Erst nach zwei Tagen habe er sich von dieser Sache wieder einigermassen erholt. ${ }^{24}$

Es gibt im Werk von Thomas Mann weitere Äusserungen zur Mathematik, zum Beispiel im Roman Doktor Faustus. Sie zeugen auf der einen Seite von der Skepsis des Geisteswissenschafters gegenüber der Mathematik, auf der anderen Seite aber auch von einer echten, aber mit etwas Unverständnis gepaarten Bewunderung. Man darf wohl im fiktiven Erzähler Serenus Zeitblom des Romans Doktor Faustus ${ }^{25}$ ein teilweises Abbild von Thomas Mann selbst sehen. Serenus Zeitblom ist studierter Altphilologe und Lehrer in Latein, Griechisch und Geschichte. Er drückt die Zurückhaltung gegenüber der Mathematik und den Naturwissenschaften deutlich aus:

Hier kann ich [...] nicht umhin, mich im Vorübergehen an dem inneren und fast geheimnisvollen $\mathrm{Zu}$ sammenhang des altphilologischen Interesses mit einem lebendig-liebevollen Sinn für die Schönheit und Vernunftwürde des Menschen zu weiden. [...] Der Mann der naturwissenschaftlichen Realien kann wohl ein Lehrer, aber niemals in dem Sinn und Grade ein Erzieher sein, wie der Jünger der bonae litterae.

Serenus Zeitblom gesteht denn auch an anderer Stelle, dass er sich in der Schule in der Disziplin der Mathematik wenig hervorgetan habe. Adrian Leverkühn hingegen, die Hauptperson, ja Titelfigur dieses Romans und Schulfreund von Serenus Zeitblom, fühlte anders. Zeitblom berichtet, - offensichtlich etwas erstaunt darüber, dass solches überhaupt möglich ist Leverkühn habe an der Mathematik ein augenscheinliches Interesse gezeigt und über Pflicht und Nötigung hinaus Algebra getrieben. Er fährt weiter:

Allerdings nimmt ja die Mathese, als angewandte Logik, die sich dennoch im rein und hoch Abstrakten hält, eine eigentümliche Mittelstellung zwischen den humanistischen und realistischen Wissenschaften ein, und aus den Erläuterungen, die Adrian [Leverkühn]

21 Thomas Mann: Lebensabriss, 1930.

22 Golo Mann: Erinnerungen und Gedanken. Eine Jugend in Deutschland, Fischer Verlag 1986. Das Zitat von Nietzsche korrigiert nach Thomas Mann Schopenhauer, Werner Classen Verlag 1948; S. 9

23 Brief vom 7. Januar 1926. Zitiert in Inge und Walter Jens: Frau Thomas Mann, rowohlt Verlag 2003; S. 139

24 Es gehören zu diesem Vortrag noch einige Worte zur weiteren Lebensgeschichte von Alfred Pringsheim. Unmittelbar nach der Machtübernahme der Nationalsozialisten emigrierte die Familie Mann aus Deutschland. Die Pringsheims blieben in München. Als Jude verlor Alfred Prigsheim seinen Status als emiritierter Professor. Bereits im Juni 1933 wurde er gezwungen, sein Haus an der Arcisstrasse zu verkaufen, da die Partei an jener Stelle einen „Führerbau“ errichten wollte; das Haus wurde im November 1933 abgerissen. Jahrelang wurde Pringsheim die Ausreise verweigert. Schliesslich erreichte er nach schwierigen Verhandlungen, dass seine wertvolle Majolika-Sammlung in London bei Sotheby's versteigert wurde. Er und seine Frau konnten darauf 1939 unter Ablieferung des grössten Teils des Erlöses (gemäss Golo Mann rund neun Zehntel) in die Schweiz emigrieren. Die Emigration fand am 31. Oktober 1939 statt, also erst nach Beginn der Kriegshandlungen gegen Polen. Es gibt Hinweise dafür, dass Winifred Wagner sich in einer schriftliche Eingabe an Hitler für Pringsheim eingesetzt hat (siehe Hanno-Walter Kruft, l.c. S. 5.). Es waren Pringsheim in der Schweiz nur noch kurze Zeit vergönnt: er starb am 25. Juni 1941 in Zürich, nachdem er noch am 2. September 1940 seinen 90. Geburtstag feiern konnte. - Weitere Informationen sowohl zum mathematischen Werk wie auch zur Majolika-Sammlung von Alfred Pringsheim findet man in Rudolf Fritsch, Daniela Rippl: Alfred Pringsheim, in Schriften der Sudetendeutschen Akademie der Naturwissenschaften und Künste, Bd. 22, München 2001, 97-128.

25 Erschienen 1947. 
mir gesprächsweise von dem Vergnügen gab, das sie ihm bereitete, ging hervor, dass er diese Zwischenstellung zugleich als erhöht, dominierend, universell empfand, oder wie er sich ausdrückte, als „das Wahre“. [...] „Du bist ein Bärenhäuter“, sagte er damals zu mir, „das nicht zu mögen. Ordnungsbeziehungen anzuschauen ist doch schliesslich das beste. Die Ordnung ist alles. Römer dreizehn: ,Was von Gott ist, das ist geordnet." "26

Später im Roman, als Leverkühn sich überlegt, sein Theologiestudium aufzugeben, ist er versucht, zur Mathematik überzugehen, bei der er auf der Schule immer gute Unterhaltung gefunden habe. Er entscheidet sich dann aber letztlich gegen die Mathematik und wendet sich ab diesem Zeitpunkt ganz der $\mathrm{Mu}-$ sik zu. Thomas Mann kann dem Roman denn auch den Untertitel Das Leben des deutschen Tonsetzers Adrian Leverkühn geben. - Eigentlich schade, dass Leverkühn sich so entschieden hat; die Mathematik hätte die Stelle der modernen Musik in diesem Roman durchaus vollwertig ersetzen können; das Interesse der Leser allerdings hätte darunter möglicherweise etwas gelitten!

Ich will später noch in einem anderen Werk von Thomas Mann Bemerkungen zur Mathematik aufspüren, aber zuerst noch einmal zu Abraham Gotthelf Kästner, den ich am Anfang kurz zu Worte kommen liess. Seine Antrittsrede in Göttingen stand unter dem Titel Was trägt das Studium der Mathematik zur sittlichen Vervollkommnung bei. Und Kästner versammelte in seinem Vortrag einige Indizien in dieser Richtung. Etwa so:

Erstens kann keiner die Mathematik wirklich lieben, wenn er nicht zugleich die Wahrheit liebt.

Dieses schon schliesst also einige von der Wissenschaft Mathematik aus. Möglicherweise liegt hier der Grund dafür, dass es so wenige Mathematiker unter den Politikern gibt. Aber die Mathematik trägt nach Kästner auch ganz konkret zur inneren Ruhe und Ausgeglichenheit bei, denn:

Die Beschäftigung mit der Mathematik ist in [...] höherem Masse dazu geeignet, heftigere Gemütsregungen zu besänftigen; denn sie kann nur bei ausgeglichenem Gemütszustand erfolgreich betrieben werden.

Wenig später wird Kästner noch konkreter:

[Ich habe] immer geglaubt, dass jene reinste Lust, die bei wissenschaftlicher Arbeit entsteht, viel dazu beitrage, die sinnlichen Begierden wenigstens einzuschränken und die Seele von Lastern zu läutern. [...] Denjenigen [...] die um ihre Forschungen bemüht sind, bleibt keine Zeit übrig, in der sie ihren Lastern frönen können.

Wenigstens, so scheint uns Kästner hier zu sagen, tun die Mathematiker nichts Dümmeres, wenn sie sich mit ihrer Wissenschaft beschäftigen.

Interessanterweise hat Thomas Mann an einer Stelle seines Romans Der Zauberberg ${ }^{27}$ diesen Gedanken ebenfalls aufgegriffen. Sein Werk Der Zauberberg spielt in der Zeit vor dem Ersten Weltkrieg in einem mondänen Lungensanatorium in Davos, dem Berghof, wo Hofrat Behrens als Arzt seine Patienten behandelt. Nicht alle Gäste des Sanatoriums sind wirklich krank und verdienen es, Patienten genannt $\mathrm{zu}$ werden; es gibt darunter auch eine grössere Anzahl, die sich in der morbiden Atmosphäre ganz wohl fühlt und ihre langen Ferien im Berghof durchaus geniesst. So kann es nicht überraschen, dass sich einige dieser unechten Patienten über die Disziplinarordnung des Hauses hinwegsetzen: Der nächtliche Besuch eines solchen im Zimmer einer Dame, nicht seiner Dame, sondern im Zimmer einer anderen, führte schliesslich zu einem Skandal. Hofrat Behrens, der Arzt, beklagt sich gegenüber Hans Castorp, der Hauptperson des Romans, wie folgt:

„Wir haben die Analyse, wir haben die Aussprache, ja Mahlzeit! Je mehr die Rasselbande sich ausspricht, umso lüsterner wird sie. Ich predige die Mathematik. [.. .] Die Beschäftigung mit der Mathematik, sage ich, ist das beste Mittel gegen Kupidität. Staatsanwalt Paravant, der stark angefochten war, hat sich drauf geworfen, er hat es jetzt mit der Quadratur des Kreises und spürt grosse Erleichterung."

Staatsanwalt Paravant, so erfahren wir an anderer Stelle, erlag früher einmal den schweren Anfechtungen, die von der Erscheinung einer neuen Patientin, der ägyptischen Fatme, ausgegangen waren. Diese Anfechtungen hatte er nun überwunden:

Mit verdoppelter Inbrunst hatte er sich [...] der klaräugigen Göttin [Mathematik] in die Arme geworfen, von deren kalmierenden Macht der Hofrat so Sittliches zu sagen wusste, und das Problem, dem bei Tag und Nacht all sein Sinnen gehörte, an das er all jene Persistenz, die ganze sportliche Zähigkeit wandte, mit der er ehemals [.. .] die Überführung armer Sünder betrieben hatte, - war kein anderes als die Quadratur des Kreises.

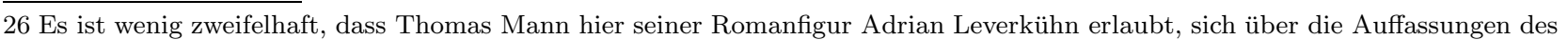
Freundes Serenus Zeitblom etwas lustig zu machen. Das Zitat aus dem Paulusbrief bezieht sich in ziemlich eindeutiger Weise nicht auf mathematisch abstrakte Ordungen in der Welt, sondern auf die hierarchische gesellschaftliche Weltordung. 27 Erschienen 1924. 


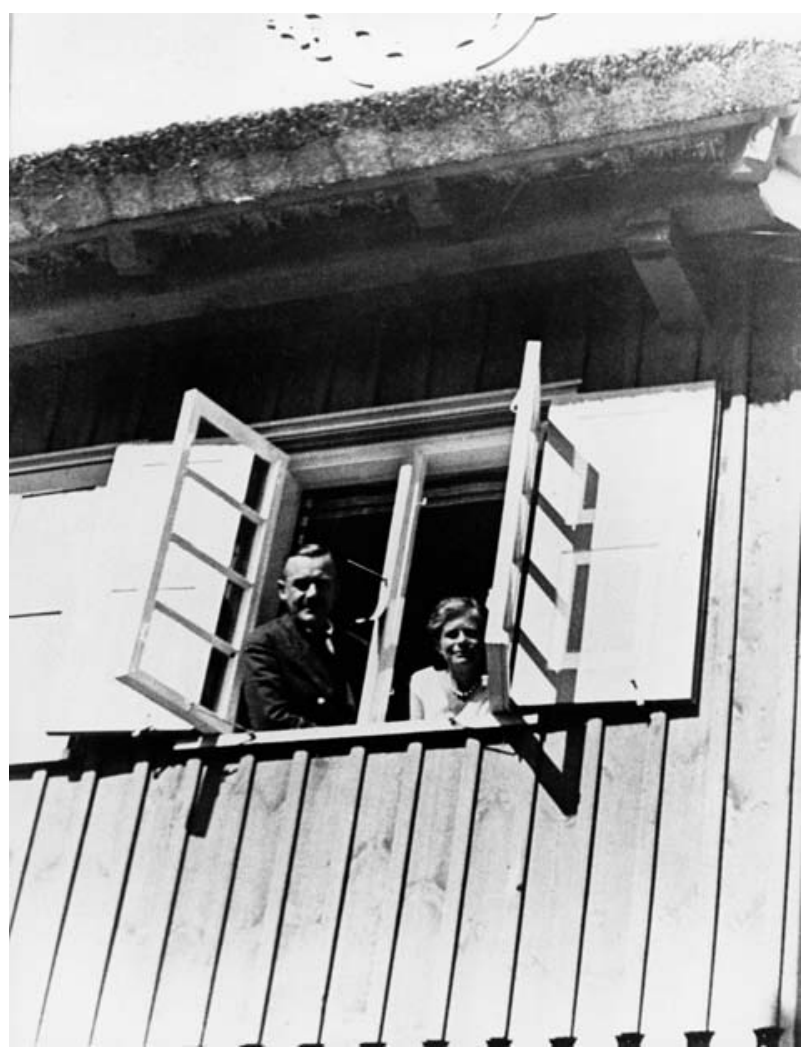

Thomas und Katia Mann im Ferienhaus Nidden, Sommer 1930 (Archiv S. Fischer Verlag)

Für das Verständnis der Fortsetzung des Textes von Thomas Mann dürften für Nichtmathematiker die folgenden Erklärungen hilfreich sein. Die Quadratur des Kreises ist ein mathematisches Problem, welches seit dem griechischen Altertum nicht nur professionelle Mathematiker sondern auch Laien immer wieder angesprochen hat. Mathematisch kann es auf Eigenschaften der Zahl $\pi$ zurückgeführt werden. Der Mathematiker Ferdinand Lindemann ${ }^{28}$ konnte 1882 zeigen, dass die Zahl $\pi$ - wie die Mathematiker sagen - transzendent ist und dass aus diesem Grund die Quadratur des Kreises mit Zirkel und Lineal nicht durchführbar ist.

Der entgleiste Beamte hatte sich im Laufe seiner Studien mit der Überzeugung durchdrungen, dass die Beweise, mit denen die Wissenschaft die Unmöglichkeit der Konstruktion erhärtet haben wollte, unstichhaltig seien und dass die planende Vorsehung ihn, Paravant, darum aus der unteren Welt der Lebendigen entfernt und hierher versetzt habe, weil sie ihn dazu ausersehen, das transzendente Ziel in den Bereich irdisch genauer Erfüllung zu reissen. So stand es mit ihm. Er zirkelte und rechnete, wo er ging und stand, bedeckte Unmassen von Papier mit Figuren, Buchstaben, Zah- len, algebraischen Symbolen, und sein gebräuntes Gesicht, das Gesicht eines scheinbar urgesunden Menschen, trug den visionären und verbissenen Ausdruck der Manie. Sein Gespräch betraf ausschliesslich und mit furchtbarer Eintönigkeit die Verhältniszahl pi [...]

Auch seriöse Mathematiker und Mathematikerinnen erkennen wohl in diesen letzten und in den folgenden Sätzen etwas von ihrem eigenen Verhalten bei ihrer mathematischen Tätigkeit.

Zuweilen glaubte Paravant sich der Offenbarung nahe. Man sah ihn öfters noch spät am Abend im verödeten und schlecht erleuchteten Speisesaal an seinem Tisch sitzen, auf dessen entblösster Platte er ein Stück Bindfaden sorgfältig in Kreisform legte, um es plötzlich, mit überrumpelnder Gebärde, zur Geraden zu strecken, danach aber, schwer aufgestützt, in bitteres Grübeln zu verfallen.

Staatsanwalt Paravant setzte sich hier in höchst laienhafter Weise mit einem längst gelösten mathematischen Problem auseinander. Obschon nur laienhaft und etwas skurril, die Beschäftigung mit Mathematik hatte bei ihm trotzdem den Effekt, den Kästner vorausgesehen und behauptet hatte: Staatsanwalt Paravant wurde ganz offensichtlich während dieser Zeit davon abgehalten, seinen Lastern zu frönen!

Nach diesen zum Teil geradezu frivolen Bemerkungen zur Mathematik von aussen wollen wir jetzt für einige Minuten ernsthaft werden, und einmal sehen, was denn die Mathematiker selber zu ihrem Fach zu sagen haben. Hier ist es ganz interessant festzustellen, dass die Ansichten über eine sehr lange Zeit hinweg in ihrer Essenz gleich oder wenigstens sehr ähnlich lauten. Zustimmen können zum Beispiel sozusagen alle der Aussage, dass in der Mathematik ein Experiment des menschlichen Geistes vorliegt, in welchem dieser seine eigenen gedanklichen Mittel bis an die Grenzen ihrer Beanspruchung erprobt. Allerdings würden wohl alle Mathematiker noch einen Zusatz machen wollen. Sie würden feststellen, dass die Wissenschaft Mathematik nicht nur ein Experiment des menschlichen Geistes ist, sondern dass sie auch weitere wesentliche Rollen erfüllt. Der Mathematik werden dabei an verschiedenen Stellen etwas unterschiedliche Rollen zugedacht, und deren Gewichtung ist nicht überall die gleiche. Aber wenn Mathematiker über Mathematik sprechen, so ist doch in überraschender Konstanz immer von den folgenden drei in irgend einer Variation die Rede. Wie in diesem Vortrag üblich will ich diese drei Rollen durch drei Zitate charakterisieren und der Dramatik halber, will ich dabei auch drei verschiedene Mathematiker zu Worte kommen lassen.

28 Es ist interessant festzustellen, dass Katia Mann im Sommersemester 1904 an der Universität München bei Ferdinand Lindemann eine Vorlesung gehört hat.

29 Hermann Weyl, 1885-1955. Das (im Original englische) Zitat stammt aus Gesammelte Abhandlungen, Springer 1988 ; S. 1. 
Die Mathematik setzt den Standard objektiver Wahrheit für jedes intellektuelle Unternehmen. ${ }^{29}$

Hermann Weyl

Dieser erste Zusatz widerspiegelt übrigens schön die Meinung des Philosophen (und Nichtmathematikers) Immanuel Kant ${ }^{30}$, der feststellte, dass in jeder besonderen Naturlehre nur soviel eigentliche Wissenschaft angetroffen werden könne, als darin Mathematik anzutreffen ist.

Zwar ist die Mathematik - wie wir oben gesehen haben - durchaus auch ein Experiment des menschlichen Geistes, ein Spiel, ein Glasperlenspiel vielleicht, aber sie ist mehr:

Mathematik ist, wie die Künste, eine freie Schöpfung des Geistes und damit Teil unserer kulturellen Tradition. $^{31}$

Beno Eckmann

Ein Teil also der kulturellen Tradition, die an die nächste Generation weiterzugeben ist, genau wie die Kunstwerke in den Museen, wie die Werke der Literatur und die Werke der Musik. - Und schliesslich, drittens, greift die Mathematik durch ihre vielfältigen Anwendungen direkt oder mittelbar über die Physik und die Technik ins tägliche Leben von uns allen ein:

Unter allen Wissenschaften ist kaum eine, die in Richtung allseitiger Verwendbarkeit eine grössere Bedeutung beanspruchen könnte, als die Mathematik. ${ }^{32}$

Felix Klein

Hermann Weyl weist in diesem Zusammenhang darauf hin, dass gerade das Faktum der mathematischnaturwissenschaftlichen Ausrichtung unsere Kultur ganz wesentlich von allen andern Kulturen der Geschichte unterscheidet. ${ }^{33}$

Es sind diese drei Dinge, die mit der Mathematik immer wieder in Verbindung gebracht werden: einmal die logische Korrektheit des Gebäudes der Mathematik, zum andern die Anerkennung der Mathematik als herausragende Leistung des menschlichen Geistes und damit als Teil der allgemeinen Kultur, und drittens die überraschende praktische und vielfältige Anwendbarkeit der abstrakten mathematischen Bildungen.

Man könnte weitere ähnliche Zitate anhäufen, und das Hohelied der Mathematik weiter singen, das da

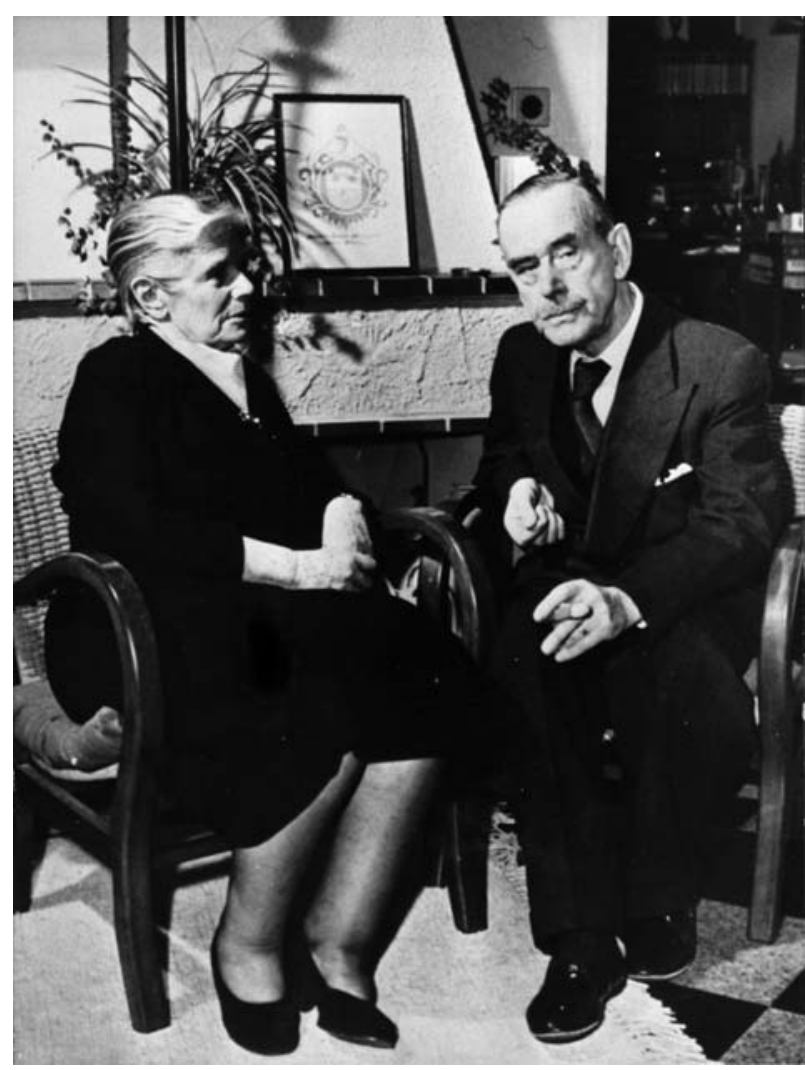

Thomas und Katia Mann 1953 (Archiv S. Fischer Verlag)

lautet Die Mathematik ist eine gar herrliche Wissenschaft. Aber ich will es dabei bewenden lassen und in dieser kurzen ernsthaften Phase meines Vortrages das Titelthema verlassen. Denn ich möchte mir zum Schluss noch einmal gestatten, die Ernsthaftigkeit etwas beiseite zu schieben.

Ich habe am Anfang festgestellt, dass es ein Mathematiker nicht leicht hat, der einen Vortrag für ein gemischtes Publikum halten soll, in dem mathematisch Gebildete sich befinden und auch solche, die wie Thomas Mann und Serenus Zeitblom der Mathematik zwar wohlwollend aber doch etwas unverständig gegenüberstehen. Ich habe hier versucht, diesem Dilemma auf meine Weise aus dem Weg zu gehen, indem ich nicht ein mathematisches Thema wählte, sondern mich auf einige Aspekte der Rezeption der Mathematik in der Geisteswelt konzentrierte. Man könnte einen anderen Ausweg wählen und die Drei-

30 Immanuel Kant (1724-1804). Das Zitat stammt aus der Vorrede seines Werkes Metaphysische Anfangsgründe der Naturwissenschaft.

31 Beno Eckmann, geb. 1917. Das Zitat stammt aus Die Zukunft der Mathematik, NZZ vom 9. August 2000, S. 46.

32 Felix Klein: Über die Beziehungen der neueren Mathematik zu den Anwendungen, Antrittsrede an der Universität in Leipzig, 25. Oktober 1880; Zeitschr. math. naturwiss. Unterricht 26 (1895), 535-540.

33 Hermann Weyl: Philosophie der Mathematik und Naturwissenschaften, R. Oldenbourg, München, 1926.

34 Johann Kaspar Ulrich, geboren 1705 in Steinegg als Sohn des dortigen Landvogtes, 1725 aufgenommen ins Ministerium. Studien in Utrecht, als Schüler von Adolf Lampe, dem er später nach Bremen folgte. Ab 1730 Pfarrer in Uitikon, 1742 Diakon bei Predigern, 1745-1768 Pfarrer am Fraumünster. 
teilung der Zuhörerschaft zur Grundlage des Vortrags machen.

Diesen Weg hat in einem anderem Zusammenhang schon Johann Kaspar Ulrich beschritten. Johann Kaspar Ulrich ${ }^{34}$, war von 1745 bis 1768 Pfarrer am Fraumünster in Zürich. In seinen Predigten teilte er seine Zuhörerschaft jeweils in drei Klassen ein, in die Unbekehrten, in die Anfänger im Glauben und in die Fortgeschrittenen im Glauben. Und er wandte sich dann in seinen Predigten der Reihe nach spezifisch an die einzelnen Klassen. Man müsste in seinen Predigten nur einzelne Wörter abändern, um sie in einem Vortrag über Mathematik vor einem aus drei Klassen gemischten Publikum verwenden zu können. So "sagte" Ulrich:

An die Fortgeschrittenen, also an die fertigen Mathematiker: Der Mann der Gesicherten [sieht] einen ganzen Strom der Gaben von dem Thron der Mathematik ausgehen.

An die Anfänger, also sozusagen an die Erstsemestrigen: Euch kann ich sagen, dass es des Mathematikprofessors Freude sei, die Gaben seines Geistes auf Euer demüthiges Flehen und Anhalten von Zeit zu Zeit in Euren Herzen zu vermehren und seine süssen Verheissungen an Euch zu erfüllen. Wer da hat, dem wird gegeben werden und er wird Überflüssiges haben.

An die Unbekehrten, also an die Antimathematiker: [Sie] mögen sich ihre Armuth, ihre Dürftigkeit, ihren Mangel selbst zuschreiben. Arme, ich kann Euch mit Grund arm nennen! Die Ursache, warum ihr der Gnadengaben der Mathematik noch nicht teilhaftig worden seid, liegt in Euch selber.

Ich wollte Ihnen, meinem Publikum am heutigen Abend, eine Unterteilung in drei Klassen nicht antun, ich tat dies in den vergangenen Jahren auch den Studierenden in meinen Vorlesungen nicht an. Denn jede solche Einteilung versieht die einzelnen Klassen mit den Etiketten, die Ulrich uns so drastisch vorführt. Es war mir deshalb immer ein Bedürfnis, mich in meinen Vorlesungen an die ganze Zuhörerschaft $\mathrm{zu}$ richten und nicht nur an einen Teil davon. Ich hoffe, dass mir dies auch heute Abend gelungen ist und dass ich Sie alle mit meinen Ausführungen etwas unterhalten konnte. So nebenbei habe ich vielleicht auch begreiflich machen können, wie die Mathematik neben allem anderen, das sie vollbringt, Tore zu anderen Dingen öffnen kann, zu Dingen, die so weit von der Mathematik entfernt liegen wie Literatur und Geschichte: Die Mathematik ist - auch in dieser Beziehung - eine gar herrliche Wissenschaft.

\section{Danksagung}

Ein herzlicher Dank geht an die Mitarbeiterinnen und Mitarbeiter der Bibliothek der ETH und an die Herren Rolf Bolt und Dr. Thomas Sprecher vom Thomas Mann Archiv, von deren Hilfe ich in vielfältiger Weise profitieren konnte. Als wichtige, noch nicht genannte Quellen erwähne ich ferner gerne das Buch von Knut Radbruch: $M a-$ thematische Spuren in der Literatur, Wissenschaftliche Buchgesellschaft 2001, sowie das Manuskript von Thomas Sprecher: Das strenge Glück der Ehe. Zum autobiographischen Kern von Thomas Manns Roman Königliche Hoheit; erschienen in überarbeiteter Form als „Ehe als Erlösung?" in Thomas Sprecher (Hrsg.): Vom "Zauberberg" zum "Doktor Faustus", Vittorio Klostermann, Frankfurt am Main, 2000.

\section{Adresse des Autors}

Professor Dr. Urs Stammbach

Departement Mathematik

ETH Zentrum

Rämistraße 101

8092 Zürich

stammb@math.ethz.ch

\section{Bildnachweise}

Die Redaktion dankt Michael Bossong, S. Fischer Verlag, und Bernd Schnarr, Bildarchiv Preußischer Kulturbesitz, für die rasche und unkomplizierte Bereitstellung von Bilddateien.

Der Abdruck erfolgt mit freundlicher Genehmigung des S. Fischer Verlags (http://www. thomasmann.de), des Bildarchivs Preußischer Kulturbesitz (www.bpk-images.de) und der Digital Library der Smithsonian Institution Libraries http://smithsonianimages.si.edu/siphoto/. 